\title{
Surgical challenges and research priorities in the era of the COVID-19 pandemic: EAES membership survey
}

\author{
Marco Milone ${ }^{1} \cdot$ Francesco Maria Carrano ${ }^{2,3} \cdot$ Emina Letić $^{4} \cdot$ Andreas Shamiyeh $^{5} \cdot$ Antonello Forgione $^{6}$. \\ Bang Wool Eom ${ }^{7}$. Beat P. Müller-Stich ${ }^{8}$. Carmen Balagué Ponz ${ }^{9} \cdot$ Christos Kontovounisios $^{10} \cdot$ Daniel Preda $^{11}$. \\ Dejan Ignjatovic ${ }^{12}$. Elisa Cassinotti ${ }^{13}$. Eugenia Yiannakopoulou ${ }^{14} \cdot$ George Theodoropoulos $^{15} \cdot$ Gil Faria $^{16,17}$. \\ Luca Morelli ${ }^{18}$. Marguerite Gorter-Stam ${ }^{19}$. Sheraz Markar ${ }^{20}$. Thanjakumar Arulampalam ${ }^{21} \cdot$ Therese Velthoven $^{22}$. \\ Stavros A. Antoniou ${ }^{23} \cdot$ Nader K. Francis ${ }^{24,25}$ (B)
}

Received: 15 May 2020 / Accepted: 19 July 2020 / Published online: 4 August 2020

(c) Springer Science+Business Media, LLC, part of Springer Nature 2020

\begin{abstract}
Background Healthcare systems and general surgeons are being challenged by the current pandemic. The European Association for Endoscopic Surgery (EAES) aimed to evaluate surgeons' experiences and perspectives, to identify gaps in knowledge, to record shortcomings in resources and to register research priorities.

Methods An ad hoc web-based survey of EAES members and affiliates was developed by the EAES Research Committee. The questionnaire consisted of 69 items divided into the following sections: (I) demographics, (II) institutional burdens and management strategies, and (III) analysis of resource, knowledge, and evidence gaps. Descriptive statistics were summarized as frequencies, medians, ranges, , and interquartile ranges, as appropriate.

Results The survey took place between March 25th and April 16th with a total of 550 surgeons from 79 countries. Eighty-one percent had to postpone elective cases or suspend their practice and 35\% assumed roles not related to their primary expertise. One-fourth of respondents reported having encountered abdominal pathologies in COVID-19-positive patients, most frequently acute appendicitis (47\% of respondents). The effect of protective measures in surgical or endoscopic procedures on infected patients, the effect of endoscopic surgery on infected patients, and the infectivity of positive patients undergoing laparoscopic surgery were prioritized as knowledge gaps and research priorities.

Conclusions Perspectives and priorities of EAES members in the era of the pandemic are hereto summarized. Research evidence is urgently needed to effectively respond to challenges arisen from the pandemic.
\end{abstract}

Keywords COVID-19 $\cdot$ Pandemic $\cdot$ Laparoscopy $\cdot$ Surgical challenges

The COVID-19 pandemic has strongly influenced healthcare systems worldwide. The rapid spread of the coronavirus required adjustment and rearrangement of healthcare services within a short period of time [1]. These changes, along with public health, social, and economic factors, have greatly affected surgical practice [2].

Surgeons were called to change their practice and assume extended roles, often not related to their primary expertise.

Electronic supplementary material The online version of this article (https://doi.org/10.1007/s00464-020-07835-7) contains supplementary material, which is available to authorized users.

Nader K. Francis

nader.francis@ydh.nhs.uk

Extended author information available on the last page of the article
Furthermore, healthcare professionals have faced challenges on a social, personal, and psychological level. In this period of social introversion, the medical community was called to address these challenges according to professional and scientific principles. Among the initial abundance of empiric and often conflicting recommendations $[3,4]$, national and institutional protocols on surgical practice [5, 6], and the profound lack of research evidence, the surgical community was called to address functional, practice, and research challenges.

The European Association for Endoscopic Surgery (EAES) has identified a lack of systematic register of these challenges. The EAES Research Committee therefore aimed to evaluate the current situation as experienced by our members, to identify gaps in knowledge, to record shortcomings 
in resources and to register research priorities through a web-based survey of EAES members and affiliates.

\section{Methods}

\section{Study group}

The study group consisted of members of the EAES Research Committee, an international panel of surgeons from 10 European countries. The steering group consisted of two Committee members (EL, MM) and the Committee chair (NKF) acted as coordinator.

\section{Survey instrument}

The study group nominated candidate questions through email communication and the steering group shortlisted questions and refined the questionnaire through feedback of all Committee members.

The questionnaire consisted of 69 items (Online Appendix) divided into the following sections:

(I) Participant demographics.

(II) Burden of the pandemic in participants' institutions and management strategies/protocols.

(III) Analysis of resource, knowledge, and evidence gaps.

The survey questions were organized into closed-ended questions with unordered choices, open-ended questions, and partially closed-ended questions, as considered appropriate. To assess knowledge gaps and research priorities, a 5-point Likert scale was used, 5 indicating the highest score.

\section{Sampling plan and invitation}

The 3500 EAES members were invited to participate in the survey through:

(I) Email invitation of EAES members and affiliated individuals.

(II) The EAES Email Newsletter sent to EAES members and affiliated individuals.

(III) Social media (Facebook, Twitter).

Two separate invitations were sent on March 25th and on April 16th. Participants were informed that data are anonymized and confidential, and were provided a web link directing to the survey instrument. Consent was implied upon acceptance to participate. The survey was hosted by
SurveyMonkey, a secure web-based survey platform. Participants were required to respond to all questions for their responses to be registered.

No formal sample size calculation was performed due to time constraints and in order for the survey to be launched within reasonable time under consideration of the urgency of the situation and the need to make results made available in a timely manner.

\section{Stratification, clustering and validity}

No relevant actions were taken for the above reasons.

\section{Statistical analysis}

A tallied summary of the results for each question was generated. Data were analyzed using descriptive statistics (SPSS $\left.^{\mathrm{TM}} \mathrm{v} 20.0, \mathrm{IBM}^{\circledR}, \mathrm{US}\right)$. Frequencies were presented as $\%$ percentages and continuous variables were presented as medians, ranges, and interquartile ranges. Summary survey responses were presented regularly to the EAES Research Committee and to the EAES Executive Board to adjust its strategic plan and address priorities.

\section{Results}

\section{Sample characteristics}

The survey took place between March 25th and April 16th. A total of 550 surgeons (16\%) from 79 countries participated. The majority of participants were based in Europe (Italy, 29\%; United Kingdom, 9\%; Romania, 5\%). There was representation from most European countries, although the number of participants from individual countries was variable (median 3; range 1-149; interquartile range 1-5). Geographical distribution of surveyed surgeons is shown in Fig. 1.

Sixty-five percent reported practicing gastrointestinal surgery in general, $15 \%$ oncological surgery and $10 \%$ upper gastrointestinal surgery. More than half reported working in academic hospitals (59\%), followed by local (26\%) and private hospitals $(12 \%)$.

\section{Assessment of the current situation}

Eight percent of the surgeons surveyed, maintained normal surgical practice for both elective and emergency surgery, 58\% cancelled elective non-cancer surgery and $23 \%$ cancelled all elective surgery. One-third $(35 \%)$ of the 
Fig. 1 Geographically distribution of surveyed surgeons
- UK

- Portugal

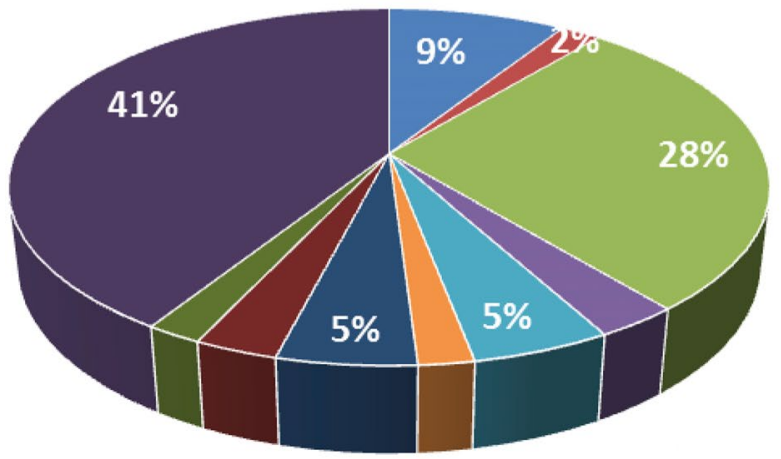

Italy

- Japan

Spain

Germany

Romania

Belgium

India

- Other countries participants have been asked to help out in other specialties; out of those, $46 \%$ were asked to help in the emergency department and $62 \%$ did not receive relevant training.

Most respondents (85\%) reported that their institution performed COVID-19 tests in patients with suspected symptoms; out of those, $61 \%$ reported that the tests were performed upon admission and $37 \%$ on the ward after admission. A dedicated ward for COVID-19-positive patients was reported by $82 \%$ to be available and COVID-19-positive surgical patients had to be admitted in such ward in $69 \%$.

Half (47\%) of the surgeons reported their hospital having treated COVID-19-positive patients with a median number of 50 (range 1-1800, interquartile range 10-160). Emergency surgery in COVID-19-positive patients was reported having been performed by 118 respondents, with a median of 3 patients (interquartile range 1-5). Out of those, $41 \%$ reported using minimally invasive surgery, with a median of 3 (range 1-43, interquartile range 1-5) (Table 1).

Sixty percent reported applying measures for minimally invasive surgery when operating on COVID-19-positive patients; $62 \%$ of those used a $\mathrm{CO}_{2}$ filter. Two-thirds (66\%) reported using standard operating procedures for COVID19-positive patients in their institution, including operatingroom-related (70\%) and perioperative care (58\%). Seventyfive percent of participants reported a reduced incidence of acute surgical conditions compared to the pre-pandemic era.

One-fourth (27\%) of respondents reported having encountered COVID-19-positive patients with abdominal pathologies, most frequently acute appendicitis (47\% of respondents). Eighteen percent reported having encountered more complications with COVID-19-positive patients, primarily respiratory ( $71 \%$ of respondents) and infective complications (18\% of respondents).

\section{Assessment of gaps}

Respondents have prioritized gaps in the area of surgical procedures and protective measures (median 4, IQR 3-5; on a 5-point Likert scale); virus-specific parameters (median 4, IQR 3-4); service redistribution (median 3, IQR 3-4); and diagnostic modalities for COVID-19-positive patients (median 3, IQR 3-4) (Fig. 2).

With regard to current gaps in resource management and protective equipment, respondents prioritized operating room protective measures (median 4, IQR 3-5), endoscopy protective measures (median 4, IQR 3-5), filtering face piece (FFP) masks (median 4, IQR 3-5) and protective materials/ equipment (median 4, IQR 3-5), own recyclable protective measures (median 4, IQR 3-5), redirection of surgical staff (median 3.5, IQR 3-5), and surgical staff training (median 3, IQR 3-4) (Fig. 3). Thirty-five percent of respondents reported enrolling COVID-19-positive surgical patients to a registry. Out of those who did not, $83 \%$ would be willing to enroll patients to an international dedicated registry.

Research gaps in the fields of the safety of surgery when using usual protective measures (median 4, IQR 3-5), the safety of minimally invasive surgery in COVID-19-positive patients (median 4, IQR 3-5), the effect and safety of pneumoperitoneum in COVID-19-positive patients (median 4, IQR 3-5), postoperative care of in COVID-19-positive patients (median 4, IQR 3-5), and the effects of conversion from minimally invasive to open surgery in COVID-19-positive patients (median 3, IQR 3-5) were prioritized (Fig. 4).

Personal psychological support was not prioritized high among respondents with a median score of 2 (IQR 1-3) (Fig. 5), whereas 68\% reported no peer support in their 
Table 1 Numbers of patients Covid-19+ treated so far

\begin{tabular}{|c|c|c|c|c|c|}
\hline \multirow[t]{2}{*}{ Question } & \multicolumn{2}{|c|}{$\begin{array}{l}\text { Surveyed } \\
\text { surgeons }\end{array}$} & \multirow[t]{2}{*}{ Total patients } & \multirow[t]{2}{*}{ Median } & \multirow[t]{2}{*}{ Geographical distribution } \\
\hline & $\mathrm{N}$ & $\%$ & & & \\
\hline Treated patients & 261 & 47 & 37,630 & $50(1-1800)$ & $\begin{array}{l}\text { Italy } 44 \% \\
\text { Spain } 28 \% \\
\text { UK } 7 \% \\
\text { Singapore } 4 \% \\
\text { Germani } 3 \% \\
\text { Philippines } 3 \% \\
\text { Belgium } 2 \% \\
\text { Portugal } 1 \% \\
\text { Netherlands } 1 \% \\
\text { Other countries with less-than-1\% } 0,2 \%\end{array}$ \\
\hline Patients in ITU & 231 & 42 & 6053 & $14(1-400)$ & $\begin{array}{l}\text { Italy } 54 \% \\
\text { Spain } 18 \% \\
\text { UK } 10 \% \\
\text { Belgium } 2 \% \\
\text { France } 2 \% \\
\text { Singapore } 2 \% \\
\text { Netherlands } 2 \% \\
\text { Germany } 1 \% \\
\text { Other countries with less-than-1\% } 1 \%\end{array}$ \\
\hline Patients underwent emergency & 118 & 21 & 1464 & $3(1-1000)$ & $\begin{array}{l}\text { Italy } 79 \% \\
\text { UK } 6 \% \\
\text { Singapore } 2 \% \\
\text { France } 1 \% \\
\text { Romania } 1 \% \\
\text { Other countries with less-than-1\% 1, 4\% }\end{array}$ \\
\hline Patients operated on using MIS & 50 & 9 & 191 & $2(1-43)$ & $\begin{array}{l}\text { Italy } 44 \% \\
\text { Spain } 30 \% \\
\text { UK } 10 \% \\
\text { Bulgaria } 5 \% \\
\text { Belgium 3\% } \\
\text { Colombia } 1 \% \\
\text { Other countries with less-than-1\% 3\% }\end{array}$ \\
\hline Patients decreased & 176 & 32 & 9317 & $8(1-3100)$ & $\begin{array}{l}\text { Indonesia } 33 \% \\
\text { Italy } 24 \% \\
\text { Ukraine } 15 \% \\
\text { UK } 14 \% \\
\text { Switzerland } 2 \% \\
\text { Poland } 1 \% \\
\text { Other countries with less-than-1\% } 0,30 \%\end{array}$ \\
\hline
\end{tabular}

institution. Psychologists to provide staff support in relation to the pandemic were reported by $57 \%$ to be available in their institution and $56 \%$ reportedly had access to this service.

\section{Discussion}

This EAES-sponsored survey found that $81 \%$ of surgeons had to postpone non-urgent cases or suspend their practice and more than one-third assumed other functional roles within their institution. Furthermore, nearly $70 \%$ reported that COVID-19-positive surgical patients had to be transferred to a dedicated ward. Among other, these findings suggest tremendous changes in institutional infrastructures and surgical practices with potential implications to the quality of surgical services.

In addition, the results of this survey suggest substantial uncertainty with regard to potential risks of surgery in infected patients and the potential effect of protective measures. Practice recommendations were issued in the early phase of the pandemic in the West; however, they lacked background research evidence, and they were conflicting or were being updated within a short period of time. 


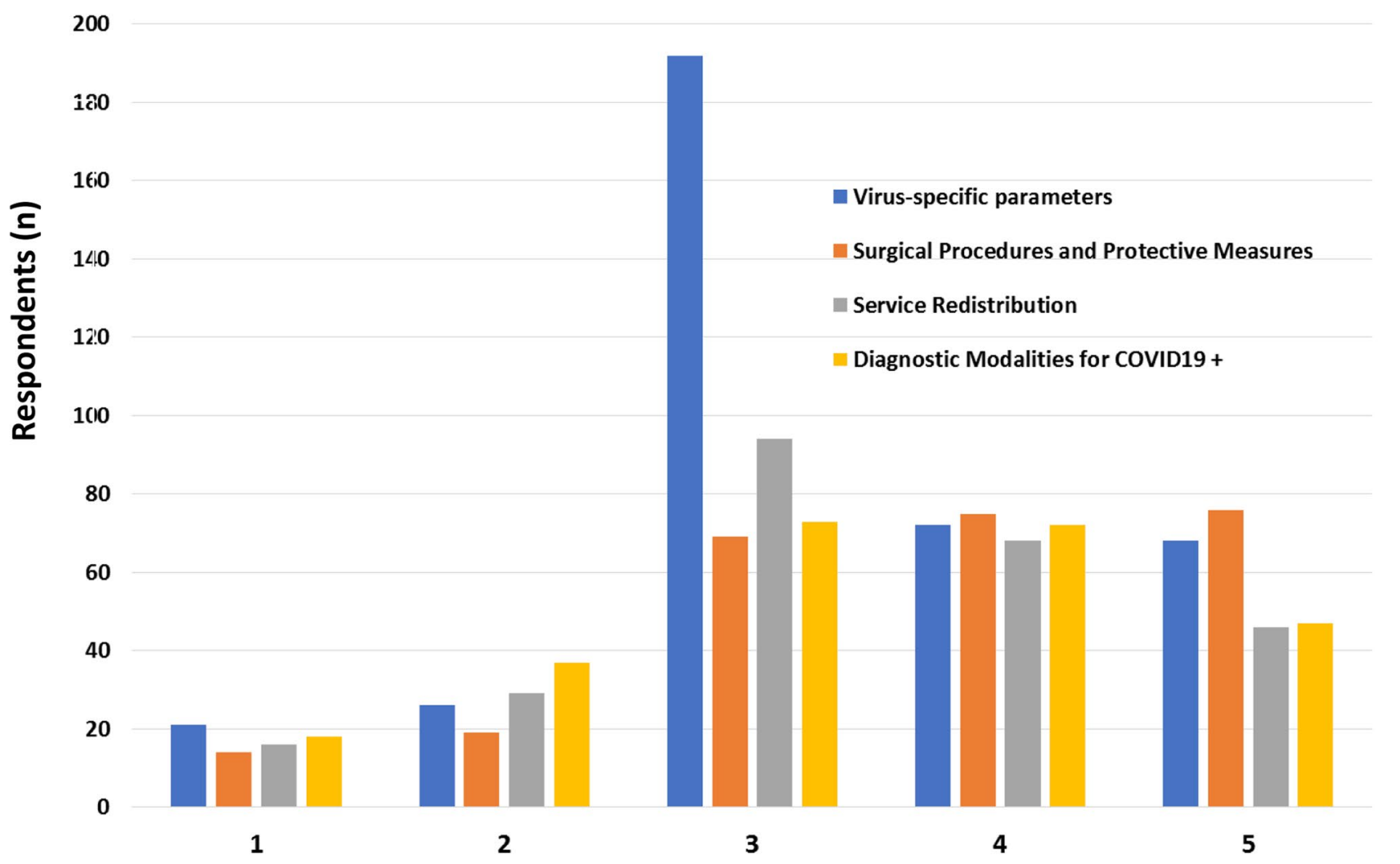

Fig. 2 Gaps prioritization (grade 1-5, 5 the maximum) in the area of surgical procedures and protective measures

Anecdotal data suggest variable directives by policymakers among European countries, based on unknown, trivial, or no background evidence supporting their putative effect. Notably, the safety of minimally invasive surgery has been questioned and directives have banned laparoscopic surgery in several countries.

Substantial uncertainty with regard to operating and endoscopy room protective measures has been documented, whereas research in this field was prioritized. Indeed, the transmission potential of COVID-19 has not been addressed by animal studies and very limited evidence refers to patients with HIV, hepatitis, SARS, and MERS. It would be probably not appropriate to extrapolate this indirect evidence and assume similar effects for SARS-CoV-2.

Rearrangement of functions among healthcare staff and the reported lack of training to serve in alternate functions may constitute a threat to the quality of healthcare services. Further emphasis on training prior to assumption of functions would be a prudent practice. The current situation suggests an urgent need to exchange information on the outcomes of surgery in infected patients.
The vast majority of respondents would be willing to provide local data to a registry; however, $65 \%$ do not provide any data resulting in waste of valuable scientific information at a time when it is critically needed. International and intersociety collaboration and promotion of existing registries would allow centralization of data for research purposes.

Survey participants have prioritized the scientific need to investigate the effect of laparoscopy and pneumoperitoneum on COVID-19-positive patients, and the postoperative management of these patients. Under consideration of the crucial need for research evidence, it would be reasonable to make registry data available to multiple research groups, allowing simultaneous conduction of exploratory analyses to address research questions.

\section{Limitations}

A limitation of this study was the response rate of $16 \%$, which may undermine generalizability and be prone to response bias. The response rate, however, is comparable with previous surgical society membership surveys [7-9]. One inherit limitations of surveys in general are recall and 
140

120

100

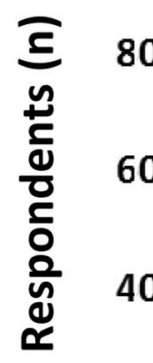

20

0

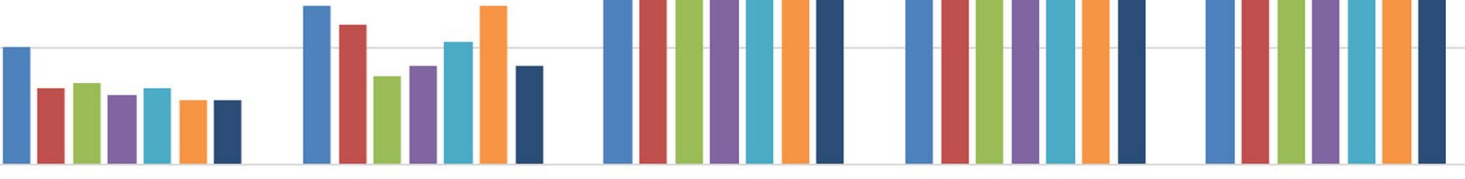

1

2

3

4

5

Redirection of surgical staff

Protective materials/equipment

On resicable protective measures
- Surgical staff training

Filtering facepiece

Endoscopy protective measures

Operative room protective measures

Fig. 3 Gaps prioritization (grade 1-5, 5 the maximum) in the area of resource management and protective equipment

selection bias of the responders, it carries the risk of the problems of personal inhibitions, indifference and unawareness of the nature of the problem which may affect the accuracy of the data.

While an argument could be made that a Delphi exercise would have been appropriate, we felt an initial broad grassroots approach would be more informative than an expert consultation. This project was developed within a few weeks in response to an urgent situation challenging surgeons on a global level and one may argue a repeat survey will be required to capture the members' views at the end of the pandemic.

\section{Conclusion}

This paper summarizes the current situation, perspectives and priorities of EAES members in the era of the COVID19 pandemic. Stepwise approach to identify and generate evidence as a response to this global challenge is a prudent strategic goal and objective of the EAES community. 


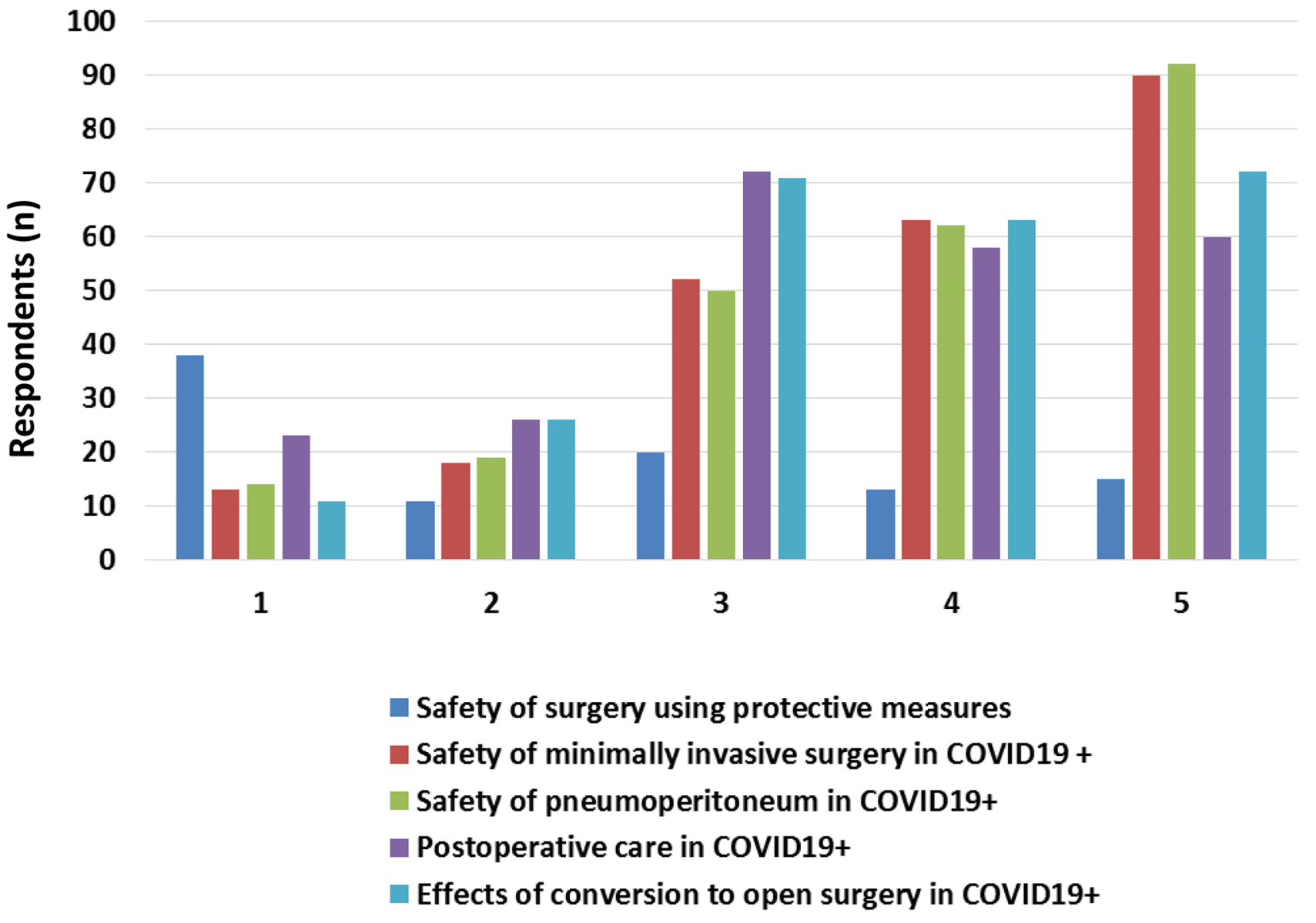

Fig. 4 Gaps prioritization (grade 1-5, 5 the maximum) in the area of the safety of surgery and minimally invasive surgery

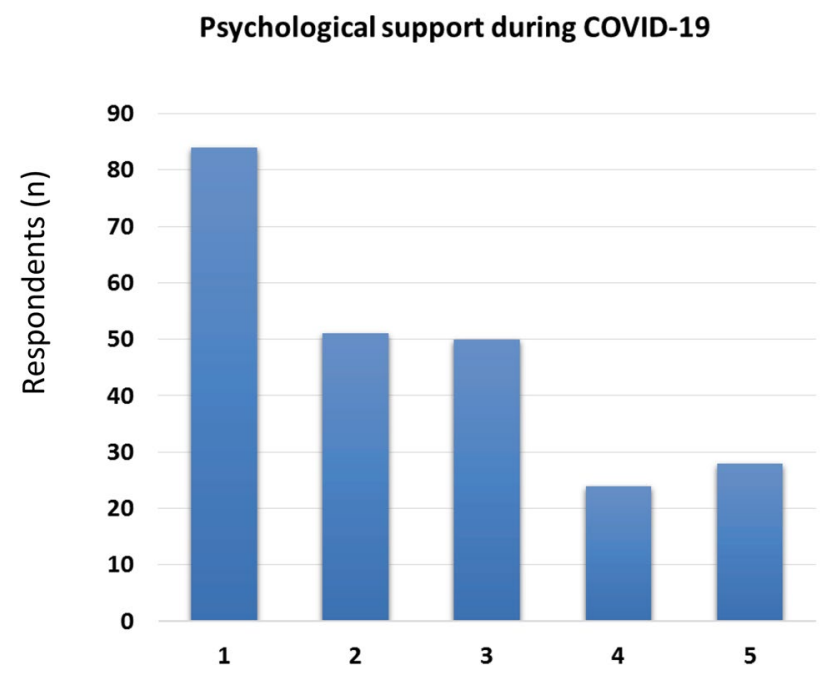

Fig. 5 Gap prioritization (grade 1-5, 5 the maximum) in the area of psychological support

Funding: None received for this study.

\section{Compliance with ethical standards}

Disclosures Marco Milone, Francesco Maria Carrano, Emina Letić, Stavros A. Antoniou, Nader K. Francis, Andreas Shamiyeh, Antonello Forgione, Bang Wool Eom, Beat Müller, Carmen Balagué Ponz, Christos Kontovounisios, Daniel Preda, Dejan Ignjatovic, Elisa Casinotti, Eugenia Yiannakopoulou, George Theodoropoulos, Gil Faria, Luca Morelli, Marguerite Gorter-Stam, Sheraz Markar, and Thanjakumar Arulampalam have no conflicts of interest or financial ties to disclose.

\section{References}

1. WHO Director-General's opening remarks at the media briefing on COVID-19. (2020) https://www.who.int/dg/speeches/detail/whodirector-general-s-opening-remarks-at-the-media-briefing-on-covid -19---11-march-2020. Accessed 11 Mar 2020

2. Di Saverio S, Pata F, Gallo G et al (2020) Coronavirus pandemic and colorectal surgery: practical advice based on the Italian experience. Colorectal Dis. https://doi.org/10.1111/codi.15056

3. Mowbray NG, Ansell J, Horwood J et al (2020) Safe management of surgical smoke in the age of COVID-19. Br J Surg. https://doi. org/10.1002/bjs.11679

4. Zheng M, Boni L, Fingerhut A (2020) Minimally invasive surgery and the novel coronavirus outbreak: lessons learned in China and Italy. Ann Surg. https://doi.org/10.1097/SLA.000000000000392 
5. Royal College of Surgeons of Edinburgh. Intercollegiate General Surgery Guidance on COVID-19, first version and following updates https://www.rcsed.ac.uk/news-public-affairs/news/2020/march/inter collegiate-general-surgery-guidance-on-covid-19-update. Accessed 5 May 2020

6. Recommendations from the Spanish Society of Surgery (AEC) v1.0 , 28 Mar 2020

7. Gorter RR, Eker HH, Gorter-Stam MA, Abis GS, Acharya A, Ankersmit $M$ et al (2016) Diagnosis and management of acute appendicitis. EAES consensus development conference 2015. Surg Endosc. https://doi.org/10.1007/s00464-016-5245-7

8. Qureshi MS, Goldsmith PJ, Maslekar S, Prasad KR, Botterill ID (2012) Synchronous resection of colorectal cancer and liver metastases: comparative views of colorectal and liver surgeons. Colorectal Dis 14(8):e477-e485

9. Keller DS, Delaney CP, Senagore AJ, Feldman LS (2016) Uptake of enhanced recovery practices by SAGES members: a survey. Surg Endosc. https://doi.org/10.1007/s00464-016-5378-8

Publisher's Note Springer Nature remains neutral with regard to jurisdictional claims in published maps and institutional affiliations.

\section{Affiliations}

\section{Marco Milone ${ }^{1}$. Francesco Maria Carrano ${ }^{2,3}$. Emina Letić ${ }^{4}$. Andreas Shamiyeh ${ }^{5}$. Antonello Forgione ${ }^{6}$. Bang Wool Eom ${ }^{7}$. Beat P. Müller-Stich ${ }^{8}$. Carmen Balagué Ponz ${ }^{9} \cdot$ Christos Kontovounisios $^{10}$ • Daniel Preda ${ }^{11}$. Dejan Ignjatovic ${ }^{12}$ - Elisa Cassinotti ${ }^{13}$. Eugenia Yiannakopoulou ${ }^{14}$. George Theodoropoulos ${ }^{15}$. Gil Faria ${ }^{16,17}$. Luca Morelli ${ }^{18}$. Marguerite Gorter-Stam ${ }^{19}$. Sheraz Markar ${ }^{20}$. Thanjakumar Arulampalam ${ }^{21}$. Therese Velthoven ${ }^{22}$. Stavros A. Antoniou ${ }^{23} \cdot$ Nader K. Francis ${ }^{24,25}$}

1 Department of Clinical Medicine and Surgery, University of Naples "Federico II", via pansini 5, Naples, Italy

2 Department of Applied Medical-Surgical Sciences, University of Rome "Tor Vergata", Rome, Italy

3 Humanitas Clinical and Research Center - IRCCS, via Manzoni 56, Rozzano, Milan, Italy

4 Department of General Surgery, Faculty of Medicine, University of Sarajevo, Čekaluša 90, 71000 Sarajevo, Bosnia and Herzegovina

5 Ludwig Boltzmann Institute for Operative Laparoscopy and 2nd Surgical Department, Academic Teaching Hospital of Linz, Krankenhausstrasse 9, 4020 Linz, Austria

6 Department of Surgical Oncology and Minimally Invasive Surgery, AIMS Academy, Niguarda Hospital, Milan, Italy

7 Center for Gastric Cancer, National Cancer Center, Research Institute and Hospital, 323 Ilsan-ro, Ilsandong-gu, Goyang, Gyeonggi-do, Republic of Korea

8 Department of General, Visceral and Transplantation Surgery, Heidelberg University Hospital, Heidelberg, Germany

9 Service of General \& Digestive Surgery, Hospital de la Santa Creu i Sant Pau, Barcelona, Spain

10 Department of Surgery and Cancer, Imperial College London, Chelsea and Westminster Campus, 369 Fulham Road, London SW10 9NH, UK

11 1st Clinic of Surgery, Craiova Emergency Clinical County Hospital, Craiova, Romania

12 Department of Digestive Surgery, Akershus University Hospital, Oslo, Norway

13 Maggiore Policlinico Hospital, Fondazione IRCCS Cà Granda, Milan, Italy
14 Department of Biomedical Sciences, Faculty of Health Sciences, University of West Attica, Athens, Greece

15 First Department of Propaedeutic Surgery, Hippocration Hospital, Medical School of Athens University, 11527 Athens, Greece

16 CINTESIS-Center for Research in Health Technologies and Information Systems, 4200-450 Porto, Portugal

17 General Surgery, Hospital de Pedro Hispano, Unidade Local de Saúde de Matosinhos, 4464-513 Senhora da Hora, Portugal

18 Department of Traslational Research and of New Surgical and Medical Technologies, Azienda Ospedaliero-Universitaria Pisana/University of Pisa, Pisa, Italy

19 Department of Surgery, VU University Medical Center, Amsterdam, The Netherlands

20 Division of Surgery, Department of Surgery \& Cancer, St Mary's Hospital - Imperial College Healthcare NHS Trust, London, UK

21 Department of General Surgery, Colchester General Hospital, Colchester, UK

22 European Association for Endoscopic Surgery, Rome, Italy

23 Department of Surgery, European University of Cyprus, Nicosia, Cyprus

24 Griffin Institute (Northwick Park Institute of Medical Research), Northwick Park Hospital, Harrow HA1 3UJ, UK

25 Yeovil District Hospital, Somerset BA21 4AT, UK 